\title{
Minimum thermal conductivity in periodically twinned $\mathrm{SrTiO}_{3}$ \\ Weixuan Li, Xiang Chen, Zexi Zheng and Youping Chen \\ Department of Mechanical and Aerospace Engineering, University of Florida, Gainesville, 32611
}

\begin{abstract}
In this work we present non-equilibrium molecular dynamics simulation results of the thermal conductivity of periodically twinned $\mathrm{SrTiO} 3$ as a function of twin boundary spacing and specimen size. We show that the thermal conductivity of $\mathrm{SrTiO} 3$ can be significantly reduced through incorporating twin boundaries. Simulation results demonstrate that the periodically twinned SrTiO3 exhibits a minimum in thermal conductivity at a critical twin spacing, and the critical twin spacing is found to increase with the size of the specimen. The mechanism underlying the minimum thermal conductivity is identified. In addition, the results of the effect of simulation cell size reveal an appreciable contribution from long wavelength phonons to the thermal conductivity of twinned SrTiO3.
\end{abstract}




\section{INTRODUCTION}

Thermoelectric materials are receiving increasing attention recent years for their potential for sustainable energy production[1]. The dimensionless figure of merit, which measures the energy conversion efficiency, is defined as $Z T=S^{2} \sigma T / k$, where the $\mathrm{S}, \sigma, \mathrm{T}$ and $\mathrm{k}$ are respectively the Seebeck coefficient, electrical conductivity, temperature and thermal conductivity. For thermoelectric materials, a $\mathrm{ZT} \geq 1$ is usually required to compete with other technologies on energy conversion [1]. Due to the high-temperature stability and environmental friendly features, ceramic oxides are extensively investigated as a type of potential thermoelectric materials. $\mathrm{SrTiO} 3$, with a cubic perovskite crystalline structure, is a potential candidate for oxide thermoelectrics[2, 3]. However, due to its relatively high thermal conductivity, $\mathrm{SrTiO}_{3}(\mathrm{STO})$ only has a low ZT value of $\sim 0.1$ [4]. In many existing works, nano-inclusion is reported to be effective to improve the thermoelectric performance of STO by reducing its electrical resistivity as well as thermal conductivity [5-7]. For further reduction of thermal conductivity, planar defects have been suggested to act as impedance to heat conduction in bulk STO[8].

Incorporating a high density of grain boundaries has been demonstrated to be able to drastically reduce the thermal conductivity of nano-grained STO 9,10$]$. However, orders of magnitude decrease of the electrical conductivity have also been observed in nanocrystalline as well as microcrystalline undoped $\mathrm{SrTiO} 3$ ceramics[11, 12]. A very special class of grain boundaries whose lattice structures are mirror images of each other in the plane of the boundary is the twin boundary, which is also known as the planar stacking fault. A periodic arrangement of twin planes forms a twinning superlattice structure. In recent years, progress has been made in fabrication of twinning superlattices with defect-free, lattice-matched, and coherent twin boundaries [13-17]. Different from the incoherent grain boundaries in nanocrystalline materials, the coherent twin boundary has been shown to be able to preserve the electrical conductivity[18-20], suggesting twinning structures to be an excellent candidate for thermoelectric applications if the thermal conductivity can be significantly reduced. While (111) twin boundaries in STO and related perovskite ceramics have been widely observed [21-24], no experimental or simulation studies of their effect on thermal conductivity have been reported, not to mention the collective role of many twin boundaries on thermal transport.

The objective of this work is to measure the thermal conductivities of periodically twinned STO and to quantify the effect of the twin spacing on the thermal conductivity using non-equilibrium molecular dynamics (MD) simulation. The remainder of the paper is organized as follows. In Section II, we introduce the structure of the coherent twin boundary in STO as well as the computer models and the simulation setup. In Section III, we present simulation results of twinned STO with varying twin spacing and specimen size, and we show that the thermal conductivity of STO can be significantly reduced by the twinning structure and that the periodically twinned SrTiO3 exhibit a minimum in thermal conductivity at a critical twin spacing; we also demonstrate the effect of the specimen size on thermal conductivity. This paper ends with a brief summary in Section IV.

\section{SIMULATION SETUP}

The open source molecular dynamics simulation software LAMMPS (Plimpton 1995) [25] is used for all the MD simulations in this work. The computer model of a periodically twinned STO is sketched in Fig. 1. The twinning structure is formed by periodical repetition of the twin lamellae A and B. The twin lamella thickness or the twin boundary spacing is denoted as $d$, and the period of the structure, denoted as $\xi$, is twice of the twin spacing $d$.

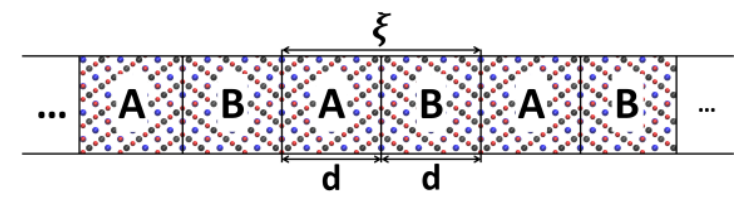

FIG. 1. Schematic illustration of the computer model for periodically twinned STO formed by repetition of twin lamellae A and $B$. The period $\xi$ is twice of the twin spacing $d$.

Among various interatomic potentials developed for STO, the rigid-ion potential for STO developed by Thomas et al. [26], described in Eq.(1), has been reported to produce grain boundary structures and energy closest to the DFTLDA results[27]. This potential has also been reported to be able to reproduce the lattice parameters with error less than $1 \%$ and thermal expansion, heat capacity with error less than 6\%[26]. In addition, MD simulations performed by Schie et al [28] showed good agreement with experimental data of heat capacity and thermal expansion for a wide temperature range. Thus, the rigid-ion potential for STO developed by Thomas et al. [26] is employed in this work to describe the interaction between atoms in the MD simulations. The potential includes a short-ranged BornMayer model and long-ranged Coulomb interactions. The parameters involved in the Born-Mayer interatomic potential are given in Table I.

$$
U=\sum_{i<j}\left(A_{i j} e^{-\frac{r_{i j}}{\rho_{i j}}}+\frac{1}{4 \pi \epsilon_{0}} \frac{q_{i} q_{j}}{r_{i j}}\right)
$$


TABLE I. Potential parameters and partial charges for $\mathrm{SrTiO} 3$

\begin{tabular}{rccc}
\hline \hline & $A_{i j}(\mathrm{eV})$ & $\rho_{i j}(A)$ & $q_{i}(e)$ \\
\hline $\mathrm{Sr}-\mathrm{O}$ & 1769.51 & 0.31984 & 1.84 \\
$\mathrm{Ti}-\mathrm{O}$ & 14567.4 & 0.197584 & 2.36 \\
$\mathrm{O}-\mathrm{O}$ & 6249.17 & 0.231472 & -1.40 \\
\hline \hline
\end{tabular}

In Fig. 2(a) we present the (111) $\Sigma 3$ twin boundary in STO characterized via high resolution transmission electron microscopy (HRTEM) by Kienzle et al[29]. The coherent twin boundary structure has also been reproduced by density functional study[30]. Shown in Fig. $2 b$ is the structure of the STO (111) $\Sigma 3$ twin boundary obtained by the molecular dynamics simulation in this work. It is seen that the interatomic potential employed in this work is able to reproduce the $\Sigma 3$ twin boundary structure observed in the experiment

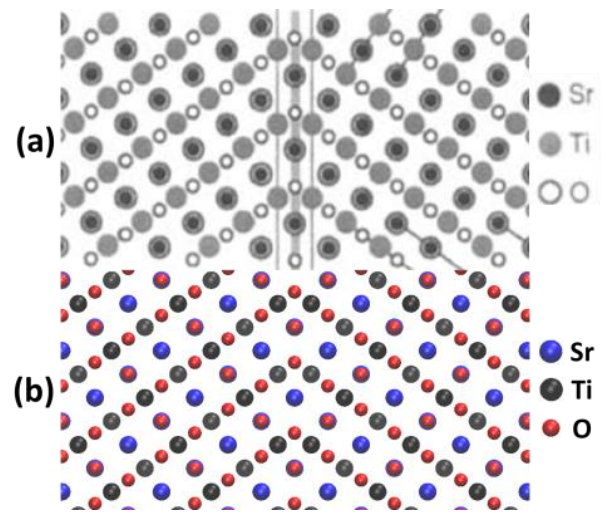

FIG. 2.(a)The $\Sigma 3 \mathrm{SrTiO} 3$ twin boundary structure observed by HRTEM (Kienzle et al. [29]); (b)The $\Sigma 3$ SrTiO3 twin boundary structure obtained by MD simulations in this work.

To quantify the effect of the twin spacing on the thermal conductivity of the twinning structure, 12 periodically twinned SrTiO3 models with different twin spacing are constructed. Each model contains 86,400 atoms and the dimensions are $5.74 \mathrm{~nm} \times 1.10 \mathrm{~nm} \times 162.32 \mathrm{~nm}$. The twin spacing and the simulation cell length of each computer model are listed in Table II.
TABLE II. Description of the computer models for investigation of the effect of twin spacing.

\begin{tabular}{ccc}
\hline \hline $\begin{array}{c}\text { Twin Spacing } \\
d(\mathrm{~nm})\end{array}$ & $\begin{array}{c}\text { Number of } \\
\text { twin lamellae }\end{array}$ & $\begin{array}{c}\text { Specimen size } \\
L(\mathrm{~nm})\end{array}$ \\
\hline 0.45 & 360 & 162.32 \\
0.68 & 240 & 162.32 \\
0.90 & 180 & 162.32 \\
1.13 & 144 & 162.32 \\
1.36 & 120 & 162.32 \\
1.81 & 90 & 162.32 \\
2.03 & 80 & 162.32 \\
2.71 & 60 & 162.32 \\
4.06 & 40 & 162.32 \\
5.41 & 30 & 162.32 \\
8.12 & 20 & 162.32 \\
16.23 & 10 & 162.32 \\
\hline \hline
\end{tabular}

The widely used nonequilibrium MD technique for thermal conductivity, i.e., the heat source-sink method, is used to simulate the steady-state heat transport and to measure the thermal conductivity of the twinned STO with various twin spacings. The time step in MD simulation is set to be $0.2 \mathrm{fs}$ and three dimensional periodic boundary conditions are employed. The initial lattice constant is chosen to be $0.3905 \mathrm{~nm}$, which is the lattice constant of STO measured in experiments at room temperature [31]. The twinned STO models are first simulated using NPT ensemble. During the NPT simulations, no appreciable volume change is observed. Based on the final structure obtained from the NPT simulation, the twinned STO models are simulated under a constant room temperature of 300 Kelvin with the volumes being fixed. Thermal transport simulation is then performed using the procedure outlined by Schelling et al.[32]. Each simulation cell is partitioned into 250 slices along the $\mathrm{z}$ direction. The regions located at the center and that at the two ends of the model serve as the heat source and sinks, respectively, as sketched in Fig. 3. A constant heat flux $q$ is then generated by adding a constant amount of energy $2 \Delta \mathrm{e}$ into the heat source region and simultaneously removing the same amount from the heat sink region at each time step. Under constant heat flux, the temperature gradient $d T / d z$ is checked for convergence over sufficient long time duration up to $3 \mathrm{~ns}$ to ensure that the system has reached steady state. Finally, the thermal conductivity is calculated according to Fourier's law: 


$$
k=-q /(d T / d z)
$$

\begin{tabular}{|c|c|c|c|}
\hline$z=-L / 2$ & $z=$ & & $z=L / 2$ \\
\hline$q$ & & $q$ & \\
\hline 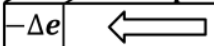 & $+2 \Delta e$ & $\Longrightarrow$ & \\
\hline
\end{tabular}

FIG. 3. Schematic representation of a three-dimensional periodic simulation cell for measurement of thermal conductivity, in which $\mathrm{L}$ is the cell length and $2 \delta$ represent the size of heat source and sink regions; $2 \Delta e$ is the amount of energy added to heat source region and removed from heat sink region at each time step; $q$ is the resulted constant heat flux.

\section{SIMULATION RESULTS AND DISCUSSIONS}

\section{A. Effect of twin spacing}

In Fig. 4, we plot the cross-plane thermal conductivity (denoted as $k_{z}$ ) as a function of the twin spacing $d$. It is seen from Fig. 4 that $k_{z}$ first decreases monotonously with increasing twin spacing $d$. After reaching the minimum value at the critical twin spacing of $d_{c}=1.81 \mathrm{~nm}, k_{z}$ then increases with the twin spacing. This behavior is similar to the experimental observation of the thermal conductivity of $\mathrm{SrTiO}_{3} / \mathrm{BaTiO}_{3}$ superlattice[33] and also to the molecular dynamics results of $\mathrm{SrTiO}_{3} / \mathrm{SrO}$ superlattice[34]. Selected temperature distributions at steady state in nano-twinned STO with different twin spacing $d$ are presented in Fig. 5. We divide the plot in Fig. 5 into three regions for discussion.

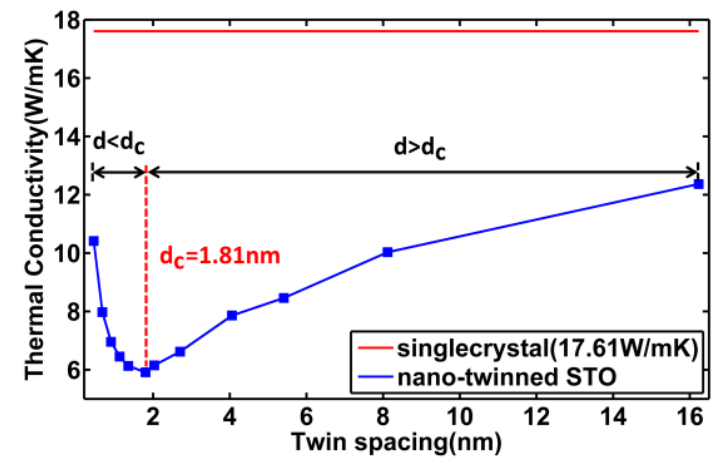

FIG. 4. Cross-plane thermal conductivity of nano-twinned STO with specimen size of $162.32 \mathrm{~nm}$.

(1) $d<d_{c}$

In this region, the temperature distribution is smooth, almost a straight line, with no temperature discontinuity at the twin boundaries being observed, as shown in Fig.5. This implies that a twinned $\mathrm{SrTiO} 3$ with small twin spacing behaves like a monolithic material for thermal transport. It is seen from Fig.4 that the thermal conductivity exhibits a decreasing tendency with increasing $d$. Similar results have been found in both natural and artificial superlattices with coherent phase interfaces [33-37]. In a theoretical calculation work by Simkin et al.[35], such phenomenon is explained by wave interference in the short-period superlattice, which causes band folding and formation of mini-bands that leads to the reduction of phonon group velocity. Our simulation results have shown an agreement with this theory. In the region $d<d_{c}$, with no temperature discontinuity at the twin boundaries being observed, the periodically twinned $\mathrm{SrTiO} 3$ behaves like a monolithic material. This implies the formation of mini-bands and band folding and hence reduced phonon group velocity. With increasing twin spacing, such zone folding effect becomes stronger, leading to reduction of phonon group velocity due to flattening of phonon dispersion curve.
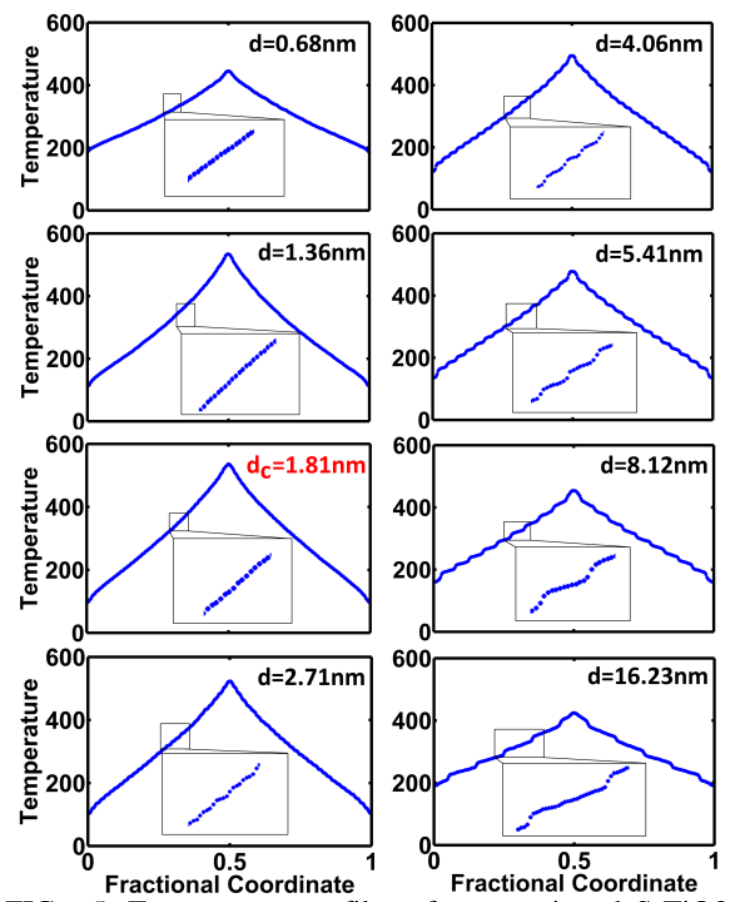

FIG. 5. Temperature profiles of nano-twinned SrTiO3 alone the fractional coordinate of the simulation with various twin spacing $d$. All the models have the same specimen size of 162.32 $\mathrm{nm}$. Inset: zoom in of temperature distributions.

\section{(2) $d>d_{c}$}

In this region, it is seen from Fig 4 that the thermal conductivity of twinned STO is positively correlated with its twin spacing. From Fig. 5 we see that with increasing twin spacing, the temperature jumps at twin boundaries become more obvious and the temperature distribution curve within the twin lamella becomes less steep. This indicates an increased contribution of the interfaces to the thermal resistance as the twin spacing becomes larger. 
To understand the mechanisms underlying the twin spacing-dependent thermal transport behavior, in Fig.6 (a) and (b), we plot the Kapitza resistance (the thermal boundary resistance) of the twin boundaries and the thermal resistivity of the twin lamella, respectively. As illustrated in Fig. 7, the Kapitza resistance is calculated by $\Delta T / q$ while the twin lamella thermal resistivity is calculated by $(\mathrm{dT} / \mathrm{dz}) / \mathrm{q}$, where $\Delta \mathrm{T}$ is the temperature jump at the twin boundaries obtained by extrapolation of the temperature curves in neighboring twins; $q$ is the heat flux and $\mathrm{dT} / \mathrm{dz}$ is the temperature gradient inside the twin lamella calculated by linear fitting. It is seen from Fig. 6 (a) and (b) that, with increasing twin spacing from $2.71 \mathrm{~nm}$ to $16.23 \mathrm{~nm}$, the Kapitza resistance of twin boundary $\left(R_{t b}\right)$ increases while the thermal resistivity of twin lamella $\left(\mathrm{r}_{\mathrm{twin}}\right)$ decreases.

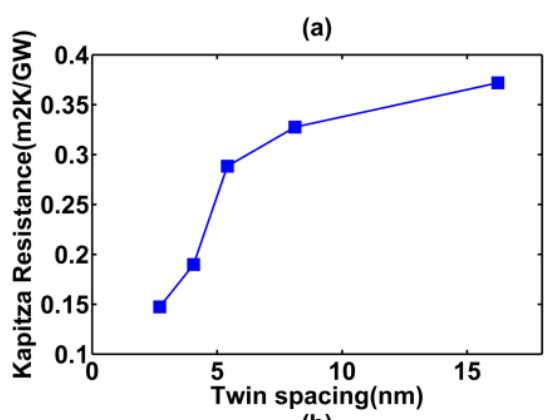

(b)

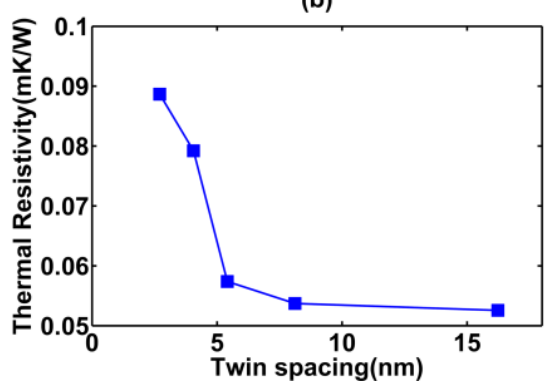

FIG. 6 (a) Kapitza resistance of twin boundaries, and (b) thermal resistivity of twin lamella.

Note that in periodically twinned STO, the phononinterface scattering which results in temperature discontinuities at the twin boundaries, and the phononphonon scattering which causes temperature gradient within the twin lamella, are two main mechanisms of thermal resistance. A comparison between the resistance of the twin boundaries and the thermal resistivity of twin lamella plotted in Fig. 6(a) and (b), respectively, suggests that these two phonon scattering mechanisms are competitive since they exhibit opposite tendency as the twin spacing increases. In addition, the non-linear temperature distributions at the vicinity of twin boundaries in Fig. 5 suggest that there is interplay between these two mechanisms. To be specific, phonons scattered back by the interface are involved in phonon-phonon scattering near twin boundaries, resulting in non-linear temperature distributions. Such effect becomes weaker as the twin spacing increases.

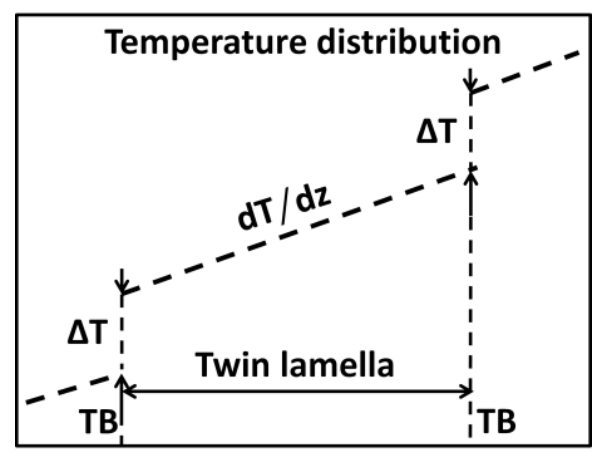

FIG.7. Illustration of how Kapitza resistance and thermal conductivity are calculated.

(3) $d=d_{c}$

At the twin spacing $d=d_{c}$, the twinned STO exhibits a minimum in the cross-plane thermal conductivity. It is seen from the temperature profiles plotted in Fig. 5 that, although the temperature drop in the nano twinned STO with $d=d_{c}$ is almost too small to see, it is at $d_{c}$ that the twin boundaries begin to cause the temperature to drop, and hence phonon-interface scattering begins to contribute to the thermal resistance. As $d$ increases, the temperature drop at twin boundaries becomes more obvious. Thus, $d=d_{c}$ is the critical twin spacing at which the thermal transport behavior of the twinned STO exhibits a transition from that of a monolithic material to that of a material containing interfaces that impede heat transfer. Note that the period of the twinned STO is twice of the critical twin spacing, i.e., $3.62 \mathrm{~nm}$. This value is within the range of reported phonon coherence lengths for STO, which is $3.0 \mathrm{~nm}$ for transverse modes and $4.5 \mathrm{~nm}$ for longitudinal modes [33]. It has been believed that there exists a coherence length of phonons in superlattices. When a superlattices period is comparable to the coherence length of the phonons, the phonon thermal transport in the superlattice is wave like; when the superlattice period is larger than the coherence length of the phonons, the phonon thermal transport is particle like. As a result of the crossover from particle like to wave like phonon transport, one observes a minimum in the thermal conductivity as a function of the superlattice period [33]. Obviously, the results of this work support the theory of phonon coherence length in superlattices, since the minimum thermal conductivity occurs at the period length of the periodically twinned STO that is comparable to the coherence length of the phonons for STO.

\section{B. Effect of specimen size}

As mentioned previously, in this work three-dimensional periodic boundary conditions are applied in the nonequilibrium molecular dynamics simulations. However, As mentioned by Termentzidis et al[38], phonons with 
wavelength larger than the size of the simulation box cannot be excited in the simulation. In addition, the thermostats scatter phonons in ways similar to those of the surface boundaries of the specimen. Thus, the effect of the size of the simulation cell reflects the effect of the specimen size, regardless whether periodic boundary conditions are applied or not. In order to investigate the size dependence of thermal conductivity of twinned STO as well as that of single crystal STO, two new sets of simulations with simulation cell length being $54.11 \mathrm{~nm}$ and $324.65 \mathrm{~nm}$, respective, and with the same loading conditions as the simulations described above are performed. The details of the computer models are listed in Table III.

TABLE III. Description of computer models for investigation of size effect.

\begin{tabular}{ccc}
\hline \hline $\begin{array}{c}\text { Twin spacing } \\
\mathrm{d}(\mathrm{nm})\end{array}$ & $\begin{array}{c}\text { Number of twin } \\
\text { lamellae }\end{array}$ & $\begin{array}{c}\text { Specimen } \\
\text { size L(nm) }\end{array}$ \\
\hline \multirow{2}{*}{0.45} & 120 & 54.11 \\
& 720 & 324.65 \\
\hline \multirow{2}{*}{0.68} & 80 & 54.11 \\
& 480 & 324.65 \\
\hline \multirow{2}{*}{0.9} & 60 & 54.11 \\
& 360 & 324.65 \\
\hline \multirow{2}{*}{1.36} & 40 & 54.11 \\
& 240 & 324.65 \\
\hline \multirow{2}{*}{1.81} & 30 & 54.11 \\
& 180 & 324.65 \\
\hline 2.03 & 160 & 324.65 \\
\hline \multirow{2}{*}{2.71} & 20 & 54.11 \\
& 120 & 324.65 \\
\hline \multirow{2}{*}{5.41} & 10 & 54.11 \\
& 60 & 324.65 \\
\hline
\end{tabular}

In Fig. 8, we plot the thermal conductivity of single crystal STO as well as that of twined STO versus the simulation cell length. It is seen from Fig. 8 that the thermal conductivity of both the single crystalline STO model and the twinned STO models are size-dependent, and the size dependence of single crystal STO is much stronger than that of twinned STO. However, the size dependence of twinned $\mathrm{SrTiO} 3$ with different twin spacing are similar.

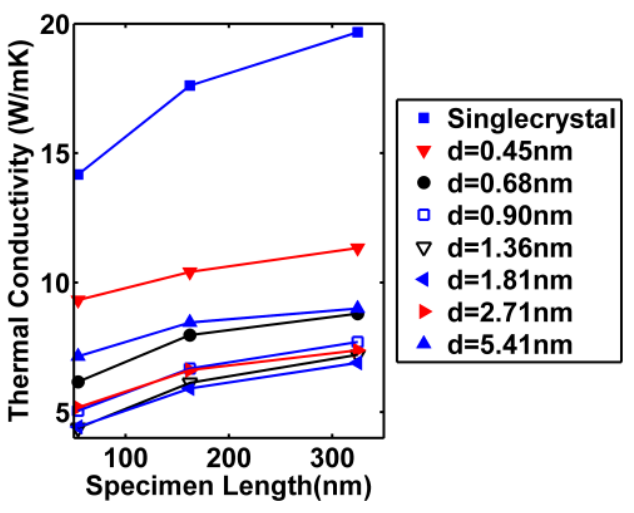

FIG. 8. Thermal conductivity of single crystalline STO and twinned STO with various twin spacings. For the purpose of comparison, all thermal conductivities are measured along <111> crystal direction.

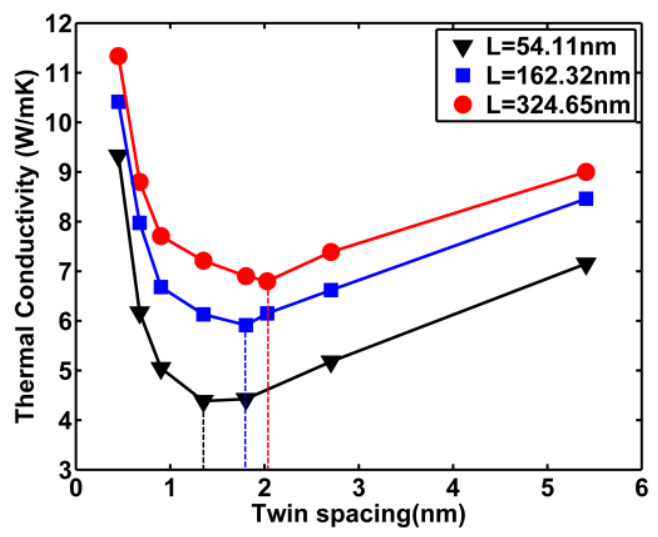

FIG. 9. Thermal conductivity of twinned STO with different specimen length $L$.

In Fig. 9, we plot the thermal conductivity of twinned STO versus twin spacing for three sets of computer models. It is seen that the critical twin spacing that produces the minimum thermal conductivity shifts from $1.36 \mathrm{~nm}$ to $2.03 \mathrm{~nm}$ from $L=65.11 \mathrm{~nm}$ to $L=324.65 \mathrm{~nm}$. The length of the simulation cell cuts off phonons whose wave lengths are larger than the size of the specimen. Since the population of long wavelength phonons increases with specimen size, leading to increased mean phonon free path and coherence length, the shift of the critical twin spacing thus indicates the change of the phonon mean free path as well as the coherence length due to the contributions from long-wavelength phonons. In addition, the increase of the thermal conductivity with simulation cell size indicates non-diffusive transport[39]. 


\section{SUMMARY AND DISCUSSION}

In this work we have computed the thermal conductivity of periodically twinned STO as a function of the twin boundary spacing. Simulation results of this work have shown that the thermal conductivity of nano-twinned STO can be reduced by $69.08 \%, 66.43 \%, 65.46 \%$, compared to that of single crystal STO, for simulation cell size of $54.11 \mathrm{~nm}, 162.32 \mathrm{~nm}$, and $324.65 \mathrm{~nm}$, respectively. Most importantly, simulation results have demonstrated the existence of minimum thermal conductivity in the periodically twinned STO.

Contrary to that in SiGe superlattices but consistent with that in STO/BTO and STO/SRO superlattices, the periodically twinned STO samples with smallest twin spacing exhibit highest thermal conductivity. As the twin spacing increases, the thermal conductivity decreases until it reaches a critical value; thereafter, the thermal conductivity increases with the twin spacing, thus producing a minimum in the thermal conductivity at a critical twin spacing. For twinned STO samples with small twin spacing, the simulation results of the temperature profiles show that there is no temperature discontinuity at the twin boundaries, indicating the thermal boundary resistance of the twinning boundaries is negligible, or the phonon-interface scattering mechanism does not make an appreciable contribution to the reduced thermal conductivity. On the other hand, for twinned STO whose twin spacing is larger than the critical twin spacing, a temperature drop at each interface can be clearly seen, indicating the role of phonon-interface scattering on the reduced thermal conductivity and demonstrating the thermal boundary resistance by the twinning boundaries. This means that for samples with larger twin spacing, both the phonon-phonon scattering and phonon-interface scattering contribute to the thermal resistance.

The simulation results of the twinned STO with different simulation cell sizes show that thermal conductivity increases with the cell size, $L$, and the critical twin spacing shift from $1.36 \mathrm{~nm}$ to $2.03 \mathrm{~nm}$ from $L=65.11 \mathrm{~nm}$ to $L=$ $324.65 \mathrm{~nm}$. The results of the effect of simulation cell size reveal an appreciable contribution from long wavelength phonons on thermal conductivity. More than $20 \%$ of thermal conductivity is from contributions of phonons whose phonon wavelengths are larger than $162.32 \mathrm{~nm}$ but smaller than $324.65 \mathrm{~nm}$.

\section{ACKNOWLEDGMENTS}

This material is based upon the work supported by the U.S. Department of Energy, Office of Basic Energy Sciences, Division of Materials Sciences and Engineering under Award \# DE-SC0006539. The work of X. C. was also supported in part by National Science Foundation under Award Numbers CMMI-1233113 and CMMI1129976.

\section{REFERENCES}

1. Kumar, S.R.S., A.Z. Barasheed, and H.N. Alshareef, High Temperature Thermoelectric Properties of Strontium Titanate Thin Films with Oxygen Vacancy and Niobium Doping. Appl. Mater. Interfaces, 2013. 5: p. 7268-7273.

2. Yang, S., N. Zhang, and Y. Chen, Concurrent atomistic-continuum simulation of polycrystalline strontium titanate. Philosophical Magazine, 2015(ahead-of-print): p. 1-20.

3. Yang, S., L. Xiong, Q. Deng, and Y. Chen, Concurrent atomistic and continuum simulation of strontium titanate. Acta Materialia, 2013. 61(1): p. 89-102.

4. Okamoto, J., G. Shimizu, S. Kubo, Y. Yamada, H. Kitagawa, A. Matushita, Y. Yamada, and F. Ishikawa, Thermoelectric properties of B-doped SrTiO3 single crystal. Journal of Physics: Conference Series, 2009. 176: p. 012042.

5. Wang, N., H. Chen, H. He, W. Norimatsu, M. Kusunoki, and K. Koumoto, Enhanced thermoelectric performance of $\mathrm{Nb}$-doped $\mathrm{SrTiO} 3$ by nano-inclusion with low thermal conductivity. Sci. Rep., 2013. 3: p. 3449.

6. Ohta, S., T. Nomura, H. Ohta, and K. Koumoto, High-temperature carrier transport and thermoelectric properties of heavily $\mathrm{La}$ - or $\mathrm{Nb}$ doped $\mathrm{SrTiO}_{3}$ single crystals. J.Appl.Phys, 2005. 97: p. 034106.

7. S.R.Popuri, A.J.M.Scott, R.A.Downie, M.A.Hall, E.Suard, R.Decourt, M.Pollet, and J.-W.G.Bos, Glass-like thermal conductivity in $\mathrm{SrTiO3}$ thermoelectrics induced by A-site vacancies. RSC Adv., 2014. 4: p. 33720.

8. Zheng, Z., X. Chen, B. Deng, A. Chernatynskiy, S. Yang, L. Xiong, and Y. Chen, Phonon thermal transport through tilt grain boundaries in strontium titanate. Journal of Applied Physics, 2014. 116(7): p. 073706.

9. Wang, Y., K. Fujinami, R. Zhang, C. Wan, N. Wang, Y. Ba, and K. Koumoto, Interfacial thermal resistance and thermal conductivity in nanograined SrTiO3. Applied Physics Express, 2010. 3(3): p. 031101.

10. Foley, B.M., H.J. Brown-Shaklee, J.C. Duda, R. Cheaito, B.J. Gibbons, D. Medlin, J.F. Ihlefeld, and P.E. Hopkins, Thermal conductivity of nanograined $\mathrm{SrTiO}_{3}$ thin films. Appl. Phys. Lett., 2012. 101: p. 231908. 
11. Abrantes, J.C.C., J.A. Labrincha, and J.R. Frade, Behavior of strontium titanate ceramics in reducing conditions suggesting enhanced conductivity along grain contacts. Journal of the European Ceramic Society, 2002. 22 (9): p. 16831691.

12. Majumdar, A., Thermoelectricity in semiconductor nanostructures. Science, 2004. 303(5659): p. 777778.

13. Xiong, S., Y.A. Kosevich, K. Sääskilahti, Y. Ni, and S. Volz, Tunable thermal conductivity in silicon twinning superlattice nanowires. Physical Review B, 2014. 90(19): p. 195439.

14. Burgess, T., S. Breuer, P. Caroff, J. Wong-Leung, Q. Gao, H.H. Tan, and C. Jagadish, Twinning superlattice formation in GaAs nanowires. ACS nano, 2013. 7(9): p. 8105-8114.

15. L.Wood, E. and F. Sansoz, Growth and properties of coherent twinning superlattice nanowires. Nanoscale, 2012. 4: p. 5268.

16. Algra, R.E., M.A. Verheijen, M.T. Borgström, L.F. Feiner, G. Immink, W.J.v. Enckevort, E. Vlieg, and E.P. Bakkers, Twinning superlattices in indium phosphide nanowires. Nature, 2008. 456(7220): p. 369-372.

17. Fissel, A., E. Bugiel, C. Wang, and H. Osten, Formation of twinning-superlattice regions by artificial stacking of Si layers. Journal of crystal growth, 2006. 290(2): p. 392-397.

18. D.L.Medlin and G.J.Snyder, Interfaces in bulk thermoelectric materials - A review for Current Opinion in Colloid and Interface Science. Current Opinion in Colloid \& Interface Science, 2009. 14: p. 226-235.

19. D.L.Medlin, Q.M.Ramasse, C.D.Spataru, and N.Y.C.Yang, Structure of the (0001) basal twin boundary in Bi2Te3. Journal of Applied Physics, 2010. 108: p. 043517.

20. L.Lu, Y.Shen, X.Chen, L.Qian, and K.Lu, Ultrahigh Strength and High Electrical Conductivity in Copper. Science, 2004. 304: p. 422.

21. Schmidt, S., D.O. Klenov, S.P. Keane, J. Lu, T.E. Mates, and S. Stemmer, Atomic structure of (111) SrTiO3 /Pt interfaces. Applied Physics Letters, 2006. 88: p. 131914.

22. M.Saylor, D., Distribution of Grain Boundaries in SrTiO3as a Function of Five Macroscopic Parameters. J. Am. Ceram. Soc., 2004. 87(4): p. 670-676.

23. Ernst, F., M.L. Mulvihill, O. Kienzle, and M. Ruhle, Preferred Grain Orientation Relationships in Sintered Perovskite Ceramics. J. Am. Ceram. Soc., 2001. 84(8): p. 1885-1890.

24. Jia, C.L., R. Rosenfeld, and K.U. A. Thust, Atomic structure of a $\Sigma=3,\{111\}$ twin-boundary junction in a BaTiO3 thin film. Philosophical Magazine Letters, 1999. 79(3): p. 99-106.

25. Plimpton, S., Fast Parallel Algorithms for ShortRange Molecular Dynamics. Journal of Computational Physics, 1995. 117(1): p. 1-19.

26. B.S.Thomas, N.A.Marks, and B.D.Begg, Developing pair potentials for simulating radiation damage in complex oxides. Nuclear Instruments and Methods in Physics Research B, 2005. 228: p. 288-292.

27. Benedek, N.A., A.L.-S. Chua, C. Elsässer, A.P. Sutton, and M.W. Finnis, Interatomic potentials for strontium titanate: An assessment of their transferability and comparison with density functional theory. Phys.Rev.B., 2008. 78: p. 064110.

28. Schie, M., A. Marchewka, T. Muller, R.A.D. Souza, and R. Waser, Molecular dynamics simulations of oxygen vacancy diffusion in SrTiO3. J.Phys.: Condens. Matter 2012. 24: p. 485002.

29. Kienzle, O. and F. Ernst, Effect of Shear Stress on the Atomistic Structure of a Grain Boundary in Strontium Titanate. J. Am. Ceram. Soc., 1997. 80(7): p. 1639-1644.

30. Hutt, S., S. Kostlmeier, and C. Elsasser, Density functional study of the Sigma3(111) [110] symmetrical tile grain boundary in SrTiO3. J. Phys.: Condens. Matter 2001. 13: p. 3949-3960.

31. Schmidbauer, M., A. Kwasniewski, and J. Schwarzkopf, High-precision absolute lattice parameter determination of $\mathrm{SrTiO} 3, \mathrm{DyScO} 3$ and NdGaO3 single crystals. Acta Cryst., 2012. B68: p. 8-14.

32. Schelling, P.K., S.R. Phillpot, and P. Keblinski, Comparison of atomic-level simulation methods for computing thermal conductivity. Phys.Rev.B., 2002. 65: p. 144306.

33. Ravichandran, J., A.K. Yadav, R. Cheaito, P.B. Rossen, A. Soukiassian, S.J. Suresha, J.C. Duda, B.M. Foley, C.-H. Lee, Y. Zhu, A.W. Lichtenberger, J.E. Moore, D.A. Muller, D.G. Schlom, P.E. Hopkins, A. Majumdar, R. Ramesh, and M.A. Zurbuchen, Crossover from incoherent to coherent phonon scattering in epitaxial oxide superlattices. Nat Mater, 2014. 13(2): p. 168-172.

34. Chernatynskiy, A., R. W.Grimes, M. A.Zurbuchen, D. R.Clarke, and S. R.Phillpot, Crossover in thermal transport properties of natural, perovskite-structured superlattices. Applied Physics Letters, 2009. 95: p. 161906.

35. Simkin, M.V. and G.D. Mahan, Minimum Thermal Conductivity of Superlattices. Physical Review Letters, 2000. 84(5): p. 927.

36. Chen, Y., D. Li, J. R.Lukes, Z. Ni, and M. Chen, Minimum superlattice thermal conductivity from 
molecular dynamics. Phys.Rev.B., 2005. 72: p. 174302.

37. Landry, E. and A. McGaughey, Effect of interfacial species mixing on phonon transport in semiconductor superlattices. Physical Review B, 2009. 79(7): p. 075316.

38. Termentzidis, K. and S. Merabia, Molecular Dynamics Simulations and Thermal Transport at the Nano-Scale, in Molecular Dynamics -

Theoretical Developments and Applications in Nanotechnology and Energy, L. Wang, Editor 2012, InTech.

39. Luckyanova, M.N., J. Garg, K. Esfarjani, A. Jandl, M.T. Bulsara, A.J. Schmidt, A.J. Minnich, S.

Chen, M.S. Dresselhaus, Z. Ren, E.A. Fitzgerald, and G. Chen, Coherent Phonon Heat Conduction in Superlattices. Science, 2012. 338: p. 936-939. 


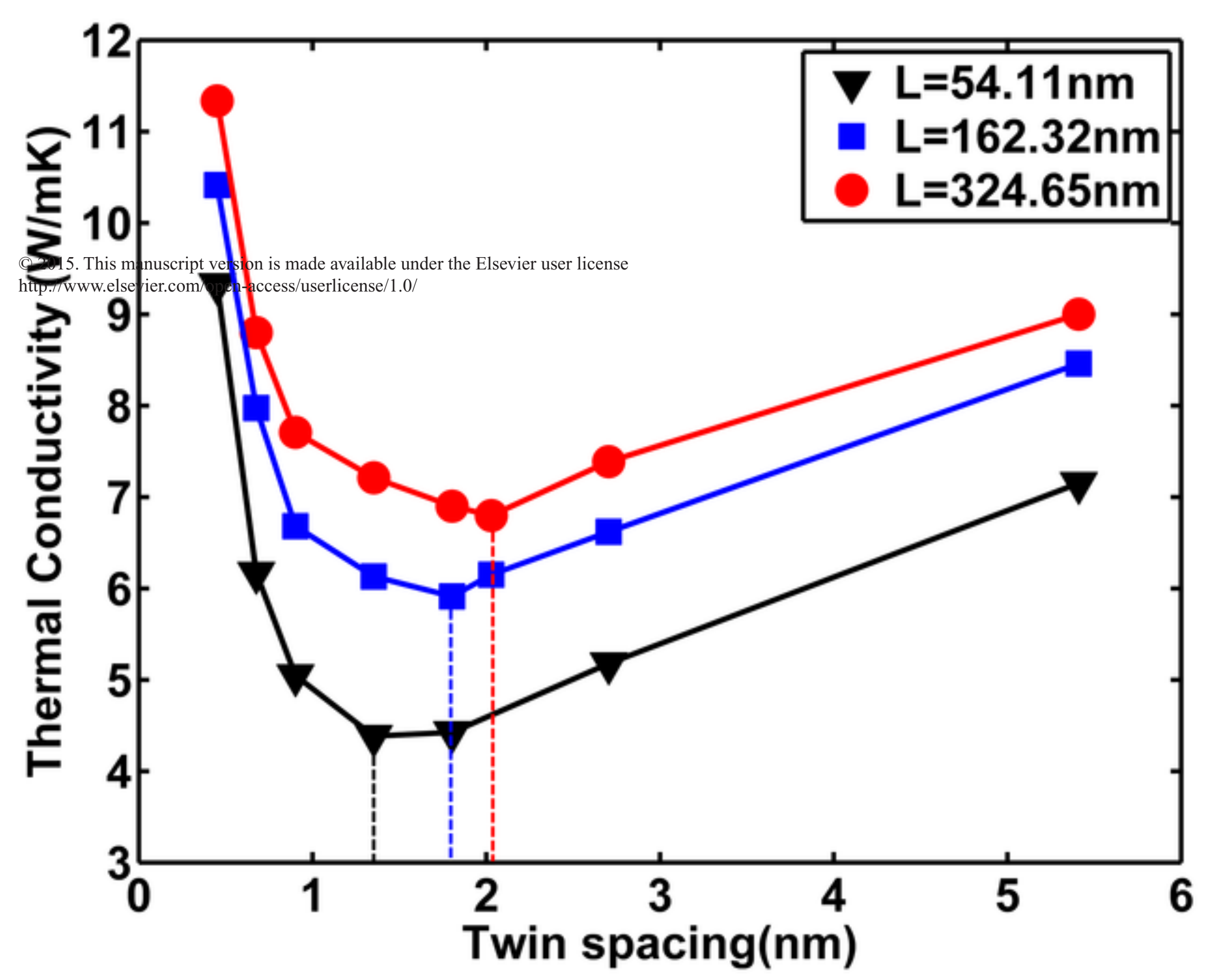

